\title{
VISCO-ELASTIC REGULARIZATION AND STRAIN SOFTENING
}

\author{
Rui S. Cardoso", , Vitor D. Silva ${ }^{\dagger}$, Humberto Varum ${ }^{*, 2}$ \\ *University of Aveiro \\ Department of Civil Engineering \\ Campus Universitário de Santiago, P-3810-193 Aveiro, Portugal \\ E-mail: ${ }^{1}$ rcardoso@civil.ua.pt \\ ${ }^{2}$ hvarum@civil.ua.pt \\ University of Coimbra \\ Department of Civil Engineering \\ Polo II da universidade-Pinhal de Marrocos, P-3030-290 Coimbra, Portugal \\ E-mail: vdsilva@dec.uc.pt
}

Keywords: Regularization, Visco-elasticity, Elasto-plasticity, Drucker-Prager, Radial Return.

\begin{abstract}
In this paper it is intended to verify the capacity of regularization of the numerical solution of an elasto-plastic problem with linear strain softening. The finite element method with a displacement approach is used. Drucker-Prager yield criteria is considered. The radial return method is used for the integration of the elasto-plastic constitutive relations. An elastovisco-plastic scheme is used to regularize the numerical solution. Two constitutive laws have been developed and implemented in a FE-program, the first represent the radial return method applied to Drucker-Prager yield criteria and the second is a time integration procedure for the Maxwell visco-elastic model. Attention is paid to finite deformations. An associative plastic flow is considered in the Drucker-Prager elasto-plastic model. The algorithms are tested in two problems with softening. Figures showing the capability of the algorithms to regularize the solution are presented.
\end{abstract}




\section{INTRODUCTION}

The use of the finite element method to solve elasto-plastic problems with strain softening behaviour, leads to the numerical collapse of the computation before the solution is obtained $[1,3,4,6,11]$. In fact the tangential global matrix ceases to be positive definite and nonuniqueness of the solution exists. This behaviour leads to pathological mesh sensitivity of the results and when modeled with finite elements where strain discontinuities arise, the plastic deformation is localized into bands of intense straining, where the band width of the localized deformations and their patterns are set by the mesh spacing $[10,11,12]$. Here, the term localization refers to situations in which the concentration of deformation into a band emerges as an outcome of the constitutive behaviour of the material and it is a characteristic feature of the inelastic deformations [11].

The purpose of the present work is to verify that the introduction of artificial viscosity in the constitutive law allows to reduce in a large scale the mesh dependency of the solution and restore the existence of a unique solution with continuous displacement $[1,3,4,6,11,14]$. The procedure is general, and therefore has the advantage of allowing the regularization of any elasto-plastic material and at the same time to model time dependent material behaviour. Furthermore, the elasto-visco-plastic constitutive equations are amenable to unconditionally stable integration. The solutions are stable and convergent with mesh refinement.

As it is usual in elastoplasticity, the Drucker-Prager model presented here is formulated in terms of rate equations [1, 4, 8, 14], which leads to an incremental formulation in the numerical integration.

The numerical integration of the elasto-plastic rate equations is carried out via a backward linear interpolation scheme, the radial return method, an unconditionally stable stress point algorithm in the hardening case $[1,4,8]$.

The visco-elastic equations are integrated by using also an implicit and unconditionally stable algorithm $[2,3,4,6]$.

This study is reported in three parts. In the first (sections 2, 3 and 4), some elementary background used in elastoplasticity, applied to Drucker-Prager criteria are summarized. The objective is to motivate the subsequent regularization procedure. In the second (section 5) the elasto-visco-plastic procedure is analyzed and in the third and last part (sections 6 and 7), some numerical examples are analyzed.

\section{BASIC CONCEPTS FOR ELASTOPLASTICITY}

\subsection{Basic equations}

As usual in elastoplasticity, material behaviour can be characterized by a set of constitutive relations of the general form $[4,6,9,14]$

$$
\begin{aligned}
& d \varepsilon_{i j}=d \varepsilon_{i j}^{e}+d \varepsilon_{i j}^{p}, \\
& d \sigma_{i j}=D_{i j k l} d \varepsilon_{i j}^{e}, \\
& F\left(\sigma_{i j}, \varepsilon_{i j}^{p}, k\right)=0, \\
& d \varepsilon_{i j}^{p}=d \lambda \frac{\partial Q}{\partial \sigma_{i j}} .
\end{aligned}
$$


Where $d \varepsilon_{i j}, d \varepsilon_{i j}^{e}$ and $d \varepsilon_{i j}^{p}$ denote the total, elastic and plastic strain incremental tensors components, $d \sigma_{i j}$ the Cauchy stress tensor components, $F$ a yield function, $k$ defines the amount of plastic deformation which depends on the plastic strain history, $d \lambda$ is the plastic multiplier, a proportionality constant, and $Q$ is the potential function.

Eq. 1 expresses the commonly assumed additive decomposition of infinitesimal strain into elastic and plastic parts. Eq. 2 establishes the elastic response (generalized Hooke's law) and is characterized by means of general linear stress-strain relations through a stiffness tensor $D_{i j k l}$. The symmetry relations $D_{i j k l}=D_{k l i j}$ are assumed. Eq. 3 represents the yield function considered. Finally, Eq. 4 expresses that the plastic strain increment is proportional to the potencial surface $Q$.

\subsection{Material stiffness matrix}

When performing the computation in an incremental displacement approach, the finite element method requires, within the increment, the calculation of the elastic material stiffness matrix or material tangential elasto-plastic stiffness matrix, depending on the load increment being elastic or elasto-plastic. The elasto-plastic tangential stiffness is a function of the stress tensor [14].

\subsubsection{Elastic stiffness matrix}

The elastic stress increment is related to the strain increment by a symmetric matrix of constants $[5,6,9,10,14]$ as follows

$$
\{d \sigma\}=[D]\{d \varepsilon\},
$$

$d \sigma_{i j}=D_{i j k l} d \varepsilon_{i j}^{e}, D_{i j k l}$ is the fourth order tensor of elasticity modulus, here assumed isotropic, $[D]$ is the material elastic stiffness and $\{d \sigma\}^{T}=\left\{d \sigma_{11}, d \sigma_{21, \cdots}, d \sigma_{33}\right\}$.

\subsubsection{Tangential elasto-plastic stiffness matrix}

The tangential elasto-plastic stiffness matrix, in incremental analysis, is defined by Eq. 6, which relates the strain increments to the stress increments

$$
\{d \sigma\}=\underbrace{\left([D]-\frac{[D]\{b\}\{a\}^{T}[D]}{\{a\}^{T}[D]\{b\}+A}\right)}_{[D]^{e p}}\{d \varepsilon\},
$$

where $[D]^{e p}$ is the elasto-plastic matrix and $A$ a parameter defined by

$$
A=-\frac{\partial F}{\partial k} \frac{d k}{d \lambda}
$$

where $k$ is a softening (hardening) parameter (section 3 ) and $d \lambda$ is the proportionality constant defined in Eq. 4, and determined as presented in sections 3 and 4. The mathematical development that leads to Eq. 6 can be found in $[6,14]$.

Since the plasticity is associative, it comes 


$$
\{a\}=\left\{\begin{array}{c}
\frac{\partial F}{\partial \sigma_{11}} \\
\vdots \\
\frac{\partial F}{\partial \sigma_{33}}
\end{array}\right\},\{b\}=\left\{\begin{array}{c}
\frac{\partial F}{\partial \sigma_{11}} \\
\vdots \\
\frac{\partial F}{\partial \sigma_{33}}
\end{array}\right\}
$$

Since de plasticity here considered is associative, $\{a\}=\{b\}$.

\section{DRUCKER-PRAGER MODEL}

For completeness, the basic equations of elastoplasticity applied to Drucker-Prager yield criteria theory are reviewed in this section.

\subsection{Drucker-Prager yield condition}

The implementation of the finite deformation plasticity model has been performed for the pressure-dependent Drucker-Prager yield criterion. The Drucker-Prager yield is defined in terms of the two first invariants of the stress tensor. More precisely, it may be defined in terms of the mean stress, $\frac{I_{1}}{3}=\sigma_{m}$ and the square root of the second invariant of the deviatoric stress component $\sqrt{J_{2}^{\prime}}=\frac{1}{3}\left(I_{1}^{2}-3 I_{2}\right)$. The elastic domain is then defined by the following expression $[6,9,12,14]$

$$
F\left(I_{1}, J_{2}^{\prime}, \sigma_{c}\right)=\alpha \frac{I_{1}}{3}+\sqrt{J_{2}^{\prime}}-k=0
$$

where

$$
\alpha=\frac{2 \sin \phi}{\sqrt{3}(3-\sin \phi)}
$$

$C$ is the cohesion, $\phi$ the angle of friction and $k$ the softening (hardening) parameter,

$$
C=\frac{k \sqrt{3}(3-\sin \phi)}{6 \cos \phi} .
$$

In principal stress space, the Drucker-Prager yield function plots as a circular cone with axis along the space diagonal as illustrated in Fig. 1.

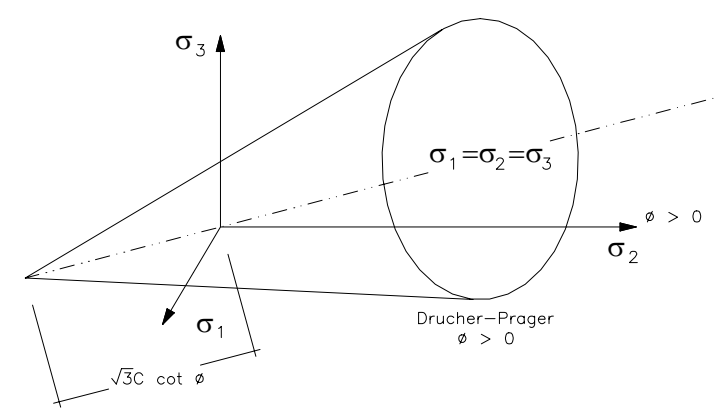

Fig.1 Drucker-Prager yield function plotted in principal stress space. 
A fundamental postulate of plasticity theory is that a stress point must remain on or within the domain defined by the yield function throughout the deformation. This requirement is expressed mathematically as

$$
F \leq 0 .
$$

Violation of Eq. 12 implies plastic material response and means that the point must be returned to the yield surface via the plastic flow rule.

\subsection{Elastic deformation}

The Hooke's law is considered for the elastic behaviour

$$
d \sigma_{i j}=D_{i j k l} d \varepsilon_{i j}^{e},
$$

where $D_{i j k l}$ can be written as

$$
D_{i j k l}=\lambda \delta_{i j} \delta_{k l}+G \delta_{i k} \delta_{j l}+G \delta_{i l} \delta_{j k},
$$

in which $\lambda$ is Lamé's constant; $G$ the shear modulus and $\delta_{i j}$ the Kronecker delta.

The isotropic and deviatoric components of the Drucker-Prager model have significantly different character, so it is worthwhile to separate the equations, which describe the elastic deformation. The superimposed dot denotes time differentiation.

$$
\{\dot{\varepsilon}\}=\frac{1}{3} \dot{\varepsilon}_{v}\{I\}+\left\{\dot{\varepsilon}^{\prime}\right\}
$$

where $\dot{\varepsilon}_{v}=$ trace $\varepsilon$ is the volumetric strain rate and $\dot{\varepsilon}^{\prime}$ the deviatoric strain rate

$$
\{\dot{\sigma}\}=\underbrace{K \dot{\varepsilon_{v}}\{I\}}_{\text {isotropic stress }}+\underbrace{2 \mathrm{G}\left\{\dot{\varepsilon}^{\prime}\right\}}_{\text {deviatoric stress }},
$$

where $\frac{1}{3}[D]\{I\}=K\{I\}$ and $[D]\left\{\dot{\varepsilon}^{\prime}\right\}=2 G\left\{\dot{\varepsilon}^{\prime}\right\}$. Thus considering a linear variation of theses quantities in the time step $\Delta t$,

$$
\{\Delta \sigma\}=K \Delta \varepsilon_{v}\{I\}+2 \mathrm{G}\left\{\Delta \varepsilon^{\prime}\right\} .
$$

\subsection{Flow rule}

During plasticity, the normality rule is applied $[1,4,6,8,9,14]$

$$
d \varepsilon_{i j}^{p}=d \lambda \frac{\partial Q}{\partial \sigma_{i j}} .
$$

Since an associative flow rule is used, $Q=F$

$$
F\left(I_{1}, J_{2}^{\prime}, k\right)=\alpha \frac{I_{1}}{3}+\sqrt{J_{2}^{\prime}}-k,
$$

substituting Eq. 19 in Eq. 18

$$
d \varepsilon_{i j}^{p}=d \lambda \frac{\partial}{\partial \sigma_{i j}}\left(\alpha \frac{I_{1}}{3}+\sqrt{J_{2}^{\prime}}-k\right) .
$$




\subsection{Softening rule}

The isotropic softening behaviour of the Drucker-Prager model is defined by the amount of plastic deformation, a linear softening rule is used. A negative value of the parameter $\theta$ leads to softening.

$$
k=k_{0}\left(1+\theta \int d \varepsilon^{p}\right),
$$

with $k$ the softening parameter (Eq. 3 and 7), and

$$
d \varepsilon^{p}=\sqrt{\frac{1}{2} d \varepsilon_{i j}^{p} d \varepsilon_{i j}^{p}}=d \lambda \sqrt{\frac{1}{2} a_{i j} a_{i j}}
$$

where

$$
d k=k_{0} \theta d \varepsilon^{p}=k_{0} \theta d \lambda \sqrt{\frac{1}{2} a_{i j} a_{i j}}
$$

so

$$
d k=k_{0} \theta d \lambda \sqrt{\frac{1}{4}+\frac{1}{6} \alpha^{2}}
$$

Since the flow rule is associate, $d \varepsilon_{i j}^{p}=\lambda a_{i j}$ with $a_{i j}=\frac{\partial F}{\partial \sigma_{i j}}$.

The mathematical development that leads to the Eq. 24 can be found in [6]. In terms of rate equation, Eq. 24 takes the following form

$$
\dot{k}=k_{0} \theta \dot{\lambda} \sqrt{\frac{1}{4}+\frac{1}{6} \alpha^{2}} \text {, with } \dot{k}=\frac{d k}{d t} \text { and } \dot{\lambda}=\frac{d \lambda}{d t} .
$$

The parameter $A$ of the global tangential elasto-plastic matrix material stiffness, Eqs. 6 and 7 , is given by

$$
\left\{\begin{array}{l}
-\frac{\partial F}{\partial k}=1 \\
d k=k_{0} \theta d \lambda \sqrt{\frac{1}{4}+\frac{1}{6} \alpha^{2}} \Rightarrow \frac{d k}{d \lambda}=k_{0} \theta \sqrt{\frac{1}{4}+\frac{1}{6} \alpha^{2}} \Rightarrow A=k_{0} \theta \sqrt{\frac{1}{4}+\frac{1}{6} \alpha^{2}} .
\end{array}\right.
$$

\section{ALGORITHM FOR THE INTEGRATION OF ELASTOPLASTIC CONSTITUTIVE RELATIONS}

For the integration of the rate equations governing the material elasto-plastic behaviour, a backward linear interpolation scheme is used. This leads to a stable and implicit algorithm, furthermore the algorithm is first order accurate. It is remarked that the accuracy and stability with which constitutive relations are integrated has a direct impact on the overall accuracy and stability of the analysis.

In this section the radial return method algorithm for Drucker-Prager model is presented. This method is equivalent to an implicit backward Euler integration also known as the closest point algorithm $[1,6,8]$.

This algorithm falls in the category of the return mapping algorithms. A return mapping algorithm can be conveniently defined based on the elastic/plastic split (Eq. 28), by first integrating the elastic equations to obtain a elastic predictor, the trial stress, which is then 
taken as an initial condition for the plastic equation. These define a plastic corrector whereby the elastically predicted stresses are relaxed onto a suitable updated yield surface.

In the context of finite element analysis, stress updates take place at the Gauss points and the incremental deformation is given. The problem to be addressed, therefore, is to update the know state variables $\varepsilon_{i}, \varepsilon_{i}^{p}, \sigma_{i}$ and $\lambda_{i}$ associated with a converged configuration $B_{i}$ into their corresponding values $\varepsilon_{i+1}, \varepsilon_{i+1}^{p}, \sigma_{i+1}$ and $\lambda_{i+1}$ on the update configuration $B_{i+1}$. In this process the incremental displacements $\Delta d_{i}$ defining the geometric updated $B_{i} \rightarrow B_{i+1}$ are assumed given.

The linear integration of a variable $X$ in the time step $\Delta t$, is calculated, solving the following equation [1]

$$
X_{n+1}=X_{n}+\left[(1-\xi) \dot{X}_{n}+\xi \dot{X}_{n+1}\right] \Delta t
$$

When $\xi=1$, Eq. 27 reduces to a implicit backward Euler integration.

As already said the isotropic and deviatoric components of the Drucker-Prager model have significantly different character, so it is worthwhile to separate the equations, which describe the plastic deformation in these two components. Considering the additive decomposition of the strain rate tensor $\{\dot{\varepsilon}\}$ in the elastic $\{\dot{\varepsilon}\}^{e}$ and the plastic components $\{\dot{\varepsilon}\}^{p}$, it becomes

$$
\{\dot{\sigma}\}=[D]\{\dot{\varepsilon}\}^{e}=[D]\left(\{\dot{\varepsilon}\}-\{\dot{\varepsilon}\}^{p}\right) .
$$

Splitting the strain rate tensor in isotropic and deviatoric components, from Eq. 28

$$
\{\dot{\varepsilon}\}=\frac{1}{3} \dot{\varepsilon}_{v}\{I\}+\left\{\dot{\varepsilon}^{\prime}\right\} \Rightarrow\{\dot{\sigma}\}=[D]\left(\frac{1}{3} \dot{\varepsilon}_{v}\{I\}+\left\{\dot{\varepsilon}^{\prime}\right\}-\{\dot{\varepsilon}\}^{p}\right) .
$$

Since

$$
\{\dot{\varepsilon}\}^{p}=\dot{\lambda}\{a\} \Rightarrow\{\dot{\varepsilon}\}^{p}=\dot{\lambda}\left(\frac{1}{2 \tau}\left\{\sigma^{\prime}\right\}+\frac{1}{3} \alpha\{I\}\right)
$$

where $\tau=\sqrt{J_{2}^{\prime}}$, substituting Eq. 30 in Eq. 29,

$$
\{\dot{\sigma}\}=[D]\left(\frac{1}{3} \dot{\varepsilon}_{v}\{I\}+\left\{\dot{\varepsilon}^{\prime}\right\}-\dot{\lambda}\left(\frac{1}{2 \tau}\left\{\sigma^{\prime}\right\}+\frac{1}{3} \alpha\{I\}\right)\right) .
$$

Taking in account the relations

$$
\frac{1}{3}[D]\{I\}=K\{I\},[D]\left\{\dot{\varepsilon}^{\prime}\right\}=2 G\left\{\dot{\varepsilon}^{\prime}\right\} \text { and }[D]\left\{\dot{\sigma}^{\prime}\right\}=2 G\left\{\dot{\sigma}^{\prime}\right\},
$$

and substituting in Eq. 31,

$$
\{\dot{\sigma}\}=\underbrace{K\left(\dot{\varepsilon}_{v}-\dot{\lambda} \alpha\right)\{I\}}_{\text {isotropic stress }}+\underbrace{2 \mathrm{G}\left\{\dot{\varepsilon}^{\prime}\right\}-\dot{\lambda} \frac{G}{\tau}\left\{\sigma^{\prime}\right\}}_{\text {deviatoric stress }} .
$$

The numerical integration of Eq. 33 is performed via a linear interpolation of $\dot{\lambda}$ and $\dot{\sigma}$, using a collocation parameter $\zeta=1$ (backward linear interpolation).

From Eq. 33 and $\frac{I_{1}}{3}=\sigma_{m}$, 


$$
K\left(\dot{\varepsilon}_{v}-\dot{\lambda} \alpha\right)\{I\}=\dot{\sigma}_{m}\{I\} \Rightarrow \dot{\sigma}_{m}=\left(\dot{\varepsilon}_{v}-\dot{\lambda} \alpha\right) K
$$

and

$$
\left\{\dot{\sigma}^{\prime}\right\}=2 \mathrm{G}\left(\left\{\dot{\varepsilon}^{\prime}\right\}-\dot{\lambda} \frac{1}{2 \tau}\left\{\sigma^{\prime}\right\}\right)
$$

considering $X=\sigma$ in Eq. 27

$$
\sigma_{n+1}=\sigma_{n}+\dot{\sigma}_{n+1} \Delta t
$$

and $X=\lambda$

$$
\{\Delta \sigma\}=\{\dot{\sigma}\}_{n+1} \Delta t \text { and } \Delta \lambda_{n+1}=\dot{\lambda}_{n+1} \Delta t .
$$

Substituting Eq.37 in Eq. 34 and 35,

$$
\begin{gathered}
\Delta \sigma_{m}=\left(\dot{\varepsilon}_{v}-\dot{\lambda} \alpha\right)_{n+1} K \Delta t \Rightarrow \Delta \sigma_{m}=K\left(\Delta \varepsilon_{v}-\Delta \lambda_{n+1} \alpha\right), \\
\left\{\Delta \sigma^{\prime}\right\}=2 \mathrm{G}\left(\left\{\dot{\varepsilon}^{\prime}\right\}-\dot{\lambda} \frac{1}{2 \tau}\left\{\sigma^{\prime}\right\}\right)_{n+1} \Delta t \Rightarrow\left\{\Delta \sigma^{\prime}\right\}=2 \mathrm{G}\left(\left\{\Delta \varepsilon^{\prime}\right\}-\Delta \lambda_{n+1} \frac{1}{2 \tau_{n+1}}\left\{\sigma^{\prime}\right\}_{n+1}\right),
\end{gathered}
$$

$K$ and $G$ represent the bulk and shear moduli, respectively, substituting Eq. 38 in Eq. 36

$$
\sigma_{m_{n+1}}=\underbrace{\sigma_{m_{n}}+K \Delta \varepsilon_{v}}_{\sigma_{m}^{t r}}-K \Delta \lambda_{n+1} \beta=\sigma_{m}^{t r}-K \alpha \Delta \lambda_{n+1},
$$

and since

$$
\left\{\sigma^{\prime}\right\}_{n+1}=\left\{\sigma^{\prime}\right\}_{n}+\left\{\Delta \sigma^{\prime}\right\}
$$

substituting Eq. 39 in Eq. 41

$$
\left\{\sigma^{\prime}\right\}_{n+1}=\underbrace{\left\{\sigma^{\prime}\right\}_{n}+2 G\left\{\Delta \varepsilon^{\prime}\right\}}_{\left\{\sigma^{\prime}\right\}^{t r}}-\frac{G}{\tau_{n+1}} \Delta \lambda_{n+1}\left\{\sigma^{\prime}\right\}_{n+1},
$$

then

$$
\left\{\begin{array}{l}
\left\{\sigma^{\prime}\right\}_{n+1}=\frac{1}{1+\frac{G}{\tau_{n+1}} \Delta \lambda_{n+1}}\left\{\sigma^{\prime}\right\}^{t r} \\
\sigma_{m_{n+1}}=\sigma_{m}^{t r}-K \alpha \Delta \lambda_{n+1} .
\end{array}\right.
$$

Since $\tau=\sqrt{J_{2}^{\prime}}$

$$
\tau_{n+1}=\frac{1}{1+\frac{G}{\tau_{n+1}} \Delta \lambda_{n+1}} \tau^{t r} \Rightarrow \tau_{n+1}=\tau^{t r}-G \Delta \lambda_{n+1} .
$$

Since Eq. 9 must be satisfied at the end of the load increment,

$$
\alpha \sigma_{m_{n+1}}+\tau_{n+1}-k_{n+1}=0 \Rightarrow \sigma_{m_{n+1}}=\frac{-\tau_{n+1}+k_{n+1}}{\alpha},
$$

from Eq. 23 


$$
\begin{aligned}
& k_{n+1}=k_{n}+k_{0} \theta \Delta \lambda_{n+1} \sqrt{\frac{1}{4}+\frac{1}{6} \alpha^{2}}, \\
& \Delta \lambda_{n+1}=\frac{\tau^{t r}-k_{n}+\alpha \sigma^{t r}}{k_{0} \theta \sqrt{\frac{1}{4}+\frac{1}{6} \alpha^{2}}+\mathrm{G}+\mathrm{K} \alpha^{2}},
\end{aligned}
$$

Eq. 40, 44 and 46 becomes

$$
\left\{\begin{array}{l}
\tau_{n+1}=\tau^{t r}-G \Delta \lambda_{n+1} \\
k_{n+1}=k_{n}+k_{0} \theta \Delta \lambda_{n+1} \sqrt{\frac{1}{4}+\frac{1}{6} \alpha^{2}} \\
\sigma_{m_{n+1}}=\frac{-\tau_{n+1}+k_{n+1}}{\alpha}
\end{array}\right.
$$

The radial return factor, in Eq. 43, can be written as a function of $k_{n+1}$

$$
\frac{1}{1+\frac{G \Delta \lambda_{n+1}}{\tau_{n+1}}}=\frac{\tau_{n+1}}{\tau_{n+1}+G \Delta \lambda_{n+1}}=\frac{\tau_{n+1}}{\tau^{t r}}=\frac{k_{n+1}-\alpha \sigma_{m_{n+1}}}{\tau^{t r}} .
$$

Eqs. (47) and (48) define the new stresses and softening parameter.

\section{ELASTO-VISCO-PLASTIC REGULARIZATION}

In order to reduce the mesh-dependency, which appears when softening takes place, an integrated visco-plastic model of the overlay type approach is considered [4], as represented in Fig. 2, in the one-dimensional case.

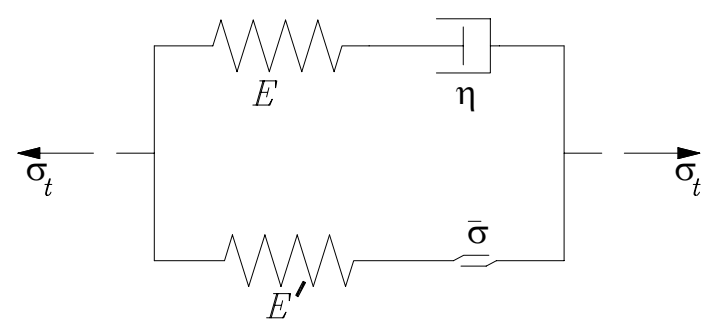

Fig. 2 Elasto-visco-plastic rheological model.

In finite element programs, the elasto-plastic constitutive law is usually implemented in a routine, in which, for a given strain increment, the stress increment and the material tangential stiffness are computed $[1,4,14]$. When a complex material behaviour is to be modelled, it is therefore convenient to use a material model of the overlay type, as the one presented in Fig. 2. In this way, stresses and stiffness can be computed separately for simpler model elements and added. The model used, is composed of an elasto-plastic element $\left(E^{\prime}, \bar{\sigma}\right)$ and a Maxwell visco-elastic element $(E, \eta)$ [5]. In its generalization to the three-dimensional case, the strain tensor is the same in both elements.

The constitutive law concerning the elasto-plastic part of the model is the one described in section 3. The time integration of the visco-elastic part (Maxwell model) is performed using an indirect approximation of the inelastic strain $[2,3,4,5,6]$. This algorithm is unconditionally stable and has a good accuracy. 
The advantages of using a positive definite matrix at each iteration step are not only economic, but also allow the computation to be extended to strain softening material or nonassociative laws [14].

The Maxwell model is composed of a serial assembly of an elastic (spring) of stiffness $E$ and a viscous element (dashpot), whose coefficient of viscosity is $\eta$. The constitutive law of the models reads

$$
\dot{\varepsilon}=\frac{\dot{\sigma}}{\eta}+\frac{\dot{\sigma}}{E}
$$

where $\sigma$ is the stress in the Maxwell model component and the dot denotes, as usually, the time derivate. Integrating this differential equation and defining $\varepsilon_{0 d}$ as the strain in the dashpot at the beginning of the time step $\Delta t$,

$$
\sigma=E\left[\varepsilon-\varepsilon_{0 d} e^{-\beta \Delta t}-\beta \int_{0}^{\Delta t} \varepsilon e^{-\beta(\Delta t-s)} d s\right] \text { with } \beta=\frac{E}{\eta},
$$

where $0 \leq s \leq \Delta t$ is the time variable. The strain in the dashpot after the time increment is then

$$
\varepsilon_{d}=\varepsilon-\frac{\sigma}{E}=\varepsilon_{0 d} e^{-\beta \Delta t}+\beta \int_{0}^{\Delta t} \varepsilon e^{-\beta(\Delta t-s)} d s .
$$

Considering a linear interpolation function for the evolution of the total strain $\varepsilon$ in the time step, $\varepsilon=\varepsilon_{0}+C s$ ( $C$ is a constant).

$$
\varepsilon_{d}=\varepsilon_{0 d} e^{-\beta \Delta t}+C \Delta t+\left(\varepsilon_{0}-\frac{C}{\beta}\right)\left(1-e^{-\beta \Delta t}\right)
$$

The stability analysis of this time integration algorithm can be found in [3].

The new stress is then

$$
\sigma_{1}=E\left(\varepsilon_{1}-\varepsilon_{d}\right) \text { with } \varepsilon_{1}=\varepsilon_{0}+C \Delta t
$$

As the constitutive law is to be used in an incremental form, the stress increment must be computed

$$
\Delta \sigma=\sigma_{1}-\sigma_{0}=E\left(\varepsilon_{1}-\varepsilon_{d}\right)-E\left(\varepsilon_{0}-\varepsilon_{0 d}\right)
$$

where $\sigma_{0}$ is the stress at the beginning of the time step. Substituting Eq. 53 in Eq. 55

$$
\Delta \sigma=-\left(1-e^{-\beta \Delta t}\right) \sigma_{0}+\frac{1-e^{-\beta \Delta t}}{\beta \Delta t} E \Delta \varepsilon
$$

with $\Delta \varepsilon=C \Delta t$.

A three-dimensional generalization of Eq. 56 is easily performed, if a constant Poisson's coefficient is used. Defining the matrix [5]

$$
[v]=\left[\begin{array}{cccccc}
1-v & v & v & 0 & 0 & 0 \\
v & 1-v & v & 0 & 0 & 0 \\
v & v & 1-v & 0 & 0 & 0 \\
0 & 0 & 0 & 1-2 v & 0 & 0 \\
0 & 0 & 0 & 0 & 1-2 v & 0 \\
0 & 0 & 0 & 0 & 0 & 1-2 v
\end{array}\right] \frac{1}{(1+v)(1-2 v)},
$$


the tensorial three-dimensional equivalent of $\Delta \sigma$ takes the form

$$
\{\Delta \sigma\}=-\left(1-e^{-\beta \Delta t}\right)\left\{\sigma_{0}\right\}+\frac{1-e^{-\beta \Delta t}}{\beta \Delta t} E[v]\{\Delta \varepsilon\} .
$$

The visco-elastic material stiffness is then

$$
\left[\frac{d \Delta \sigma}{d \Delta \varepsilon}\right]=E \frac{1-e^{-\beta \Delta t}}{\beta \Delta t}[v]
$$

\section{ELEMENTS USED IN THE FEA}

The elements used in the examples analysed in section 7 belong to the FEPS 3.3 library, [13]. All these elements are isoparametric Lagrangean type with linear and quadratic shape functions.

The concept of isoparametric element is fully described in the literature [14], and the advantages in using them have been investigated in [12].

Three types of elements, Fig. 3, where used to obtain the specimens geometry needed to perform the numerical examples described in section 7 .

First, to define the specimen geometry, the PT6E element is used. It is a pentahedric volume element with 5 faces, six nodes with three independent displacements each, and two integration points.

Second, in order to apply distributed loads in the volumetric elements, it is necessary to consider surface element in the faces of the volume elements. Triangular surface elements (TMV3 element) were considered in the specimen faces with 3 nodes, three independent displacements per node and one integration point.

Finally, in the faces of the specimen geometry with 4 nodes, the QMV4 element was adopted. It is a quadrilateral surface element with 4 nodes, three independent displacements per node and four integration points.

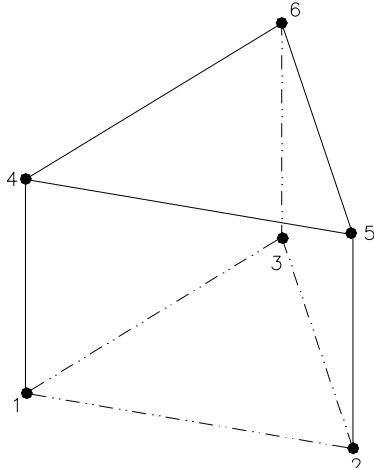

a) PT6E element

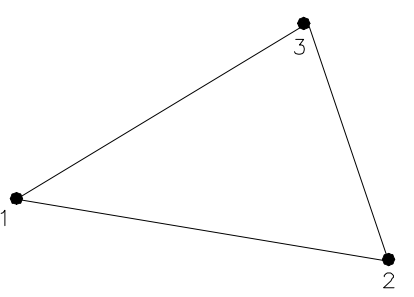

b) TMV3 element

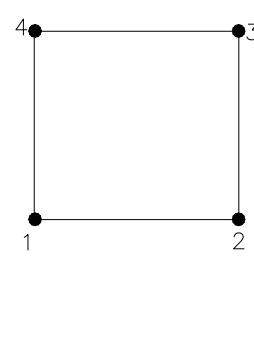

c) QMV4 element

Fig. 3 Finite elements used in the analysis.

\section{NUMERICAL EXAMPLES}

\subsection{Numerical validation of the algorithms}

The performance of the visco-elastic algorithm was tested with two numerical examples of a soil specimen. The two numerical examples studied differ on the value of the softening parameter. For the elastic perfectly plastic case $\theta=0$, Fig. 6 , and for the elasto-plastic with softening example $\theta=-150$, Fig. 5 . The specimen geometry, boundary conditions and loading history are represented in Fig. 4. To define the specimen geometry, two prismatic elements (PT6E) were used. The geometry of the soil specimen is a cube 1.0 meter dimension in each 
direction. The displacements in z-direction of points 1, 2, 5 and 6 , and $\mathrm{x}$-direction of points 1 , 2, 3 and 4 are blocked. For the Poisson's coefficient of the visco-elastic model the same value as in the elasto-plastic law $(v=0.3)$ is assumed. A constant pressure $p$ is applied in the lateral faces at first, while the imposed vertical displacement $d$ is applied in nodes 2, 3, 6 and 7 in 200 incremental steps, at diferentes rate (when viscosity is considered), as shown in Fig. 4. Loading steps of $0.06 \mathrm{~s}$ were considered. For the elasto-plastic law $\alpha=0.3, k=12 \mathrm{MPa}, E^{\prime}=$ $20600 \mathrm{MPa}$. The relaxation parameter and elastic modulus considered in the visco-elastic components are $\beta=16 \mathrm{~s}^{-1}$ and $E=20600 M P a$, respectively.
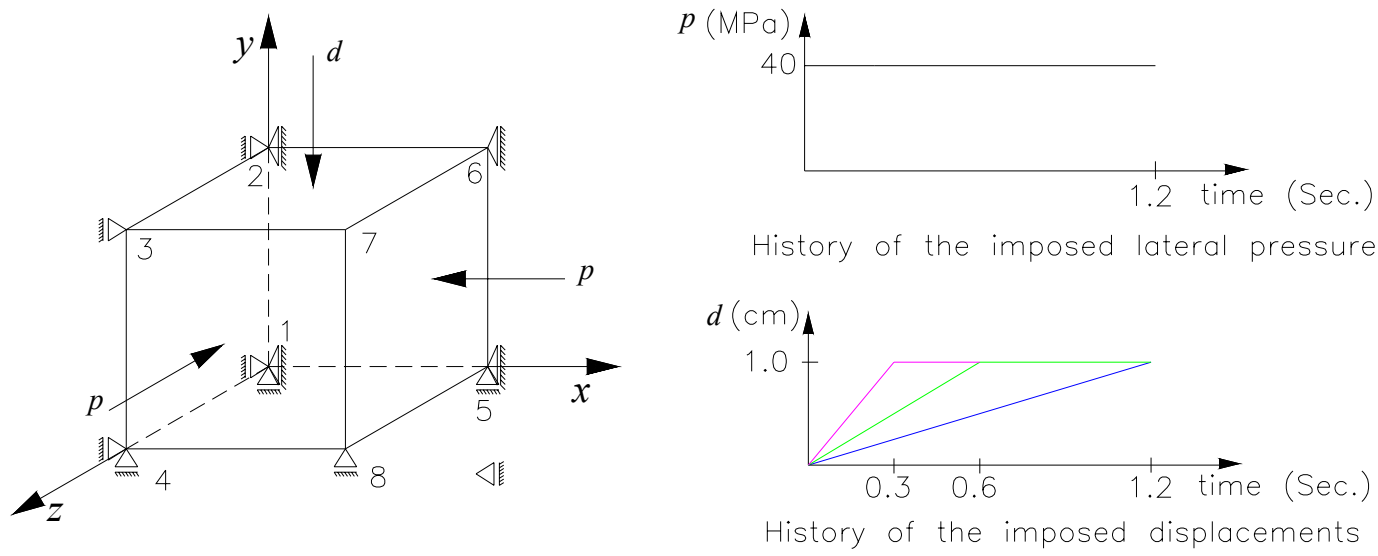

Fig. 4 Specimen geometry, boundary conditions and loading history.

In Figs. 5 and 6, the evolution of the vertical stress inside the cube, against time is plotted, three curves consider that the behaviour is elasto-visco-plastic but with diferentes rates for the imposed displacement: $8.33 \mathrm{~mm} / \mathrm{s}, 16.67 \mathrm{~mm} / \mathrm{s}$ and $33.33 \mathrm{~mm} / \mathrm{s}$, For the other curve the elastoplastic law is considered. For the rates: $16.67 \mathrm{~mm} / \mathrm{s}$ and $33.3 \mathrm{~mm} / \mathrm{s}$, the imposed displacement is maintained constant until the last step.

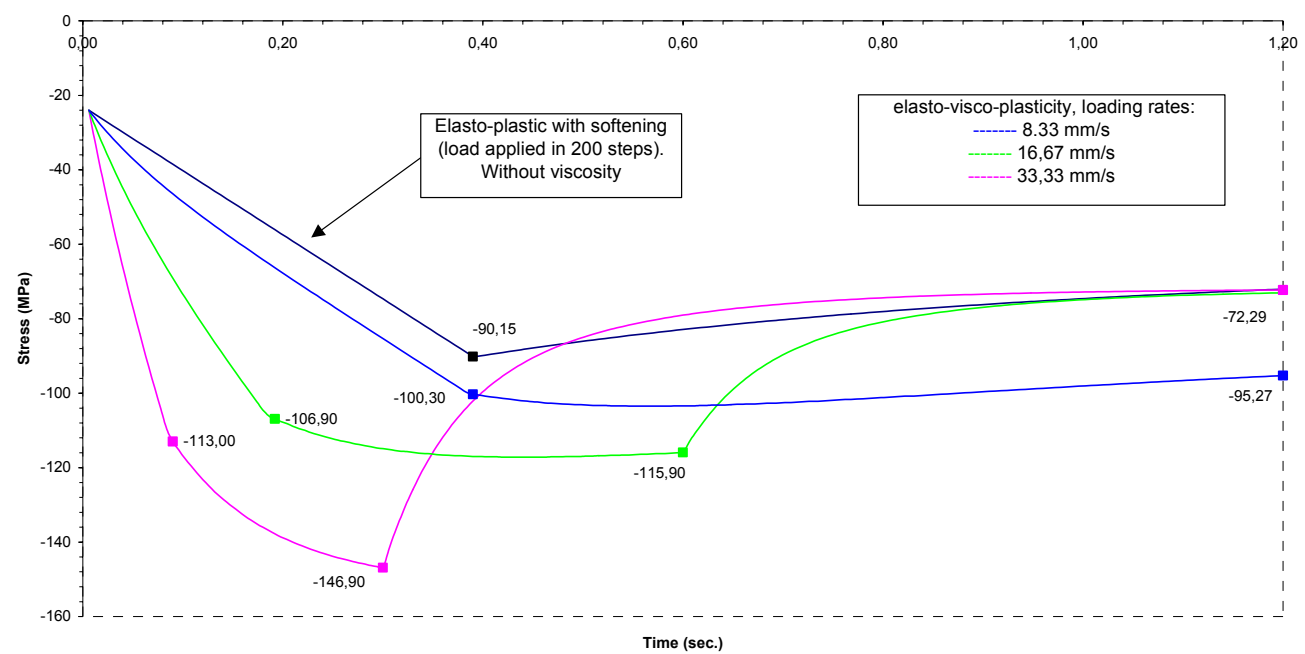

Fig. 5 Elasto-(visco)-plastic with softening. 


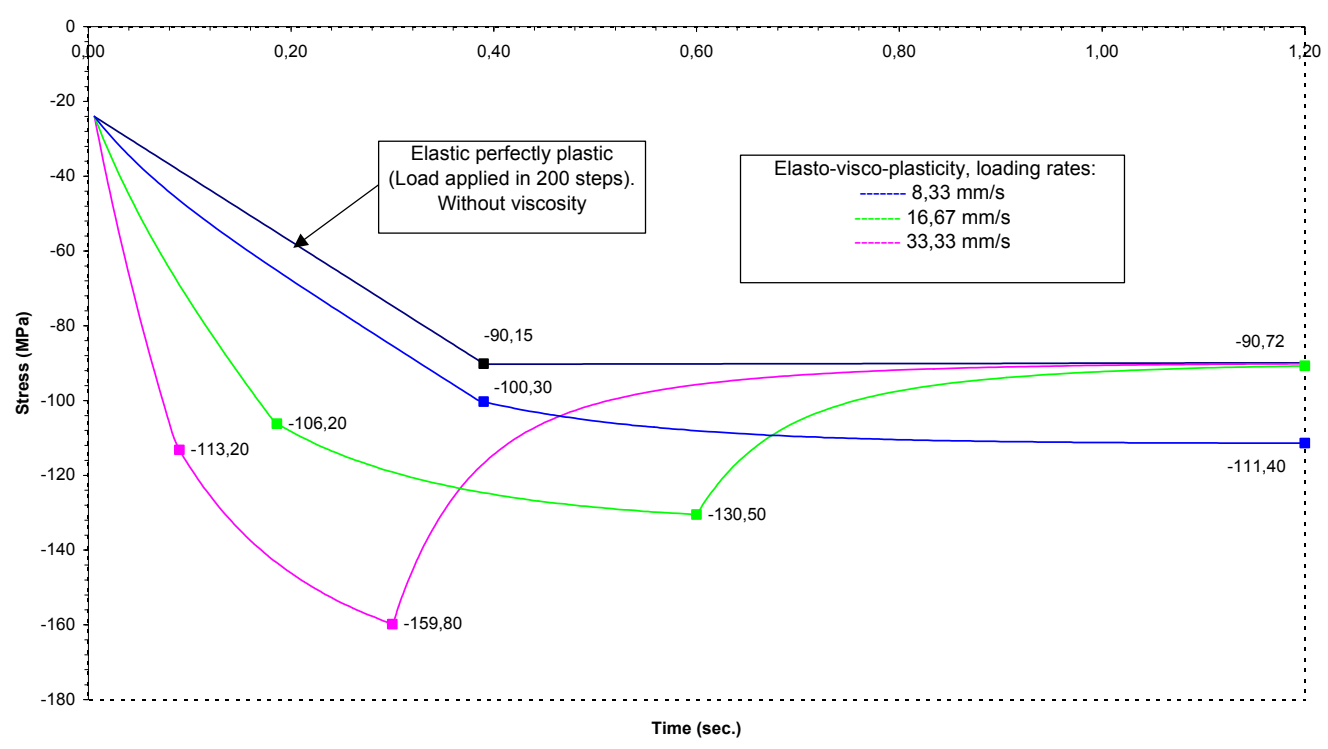

Fig. 6 Elasto-(visco)-perfectly plastic.

\subsection{Elasto-visco-plastic model}

In order to test the described elasto-visco-plastic regularization techniques in the presence of severe softening, the formation of shear bands in a soil specimen is simulated using the rheological model of Fig. 2. The model was implemented in FEPS [13], without consideration of dynamic effects and using a classical computation of elasto-plastic material stiffness.

The shear bands simulation is obtained by considering three different meshes on the same specimen. The efficiency of the regularization procedures is analyzed in the case of severe softening, the softening parameter $\theta$ is considered equal to -150 .

The specimen geometry, boundary conditions and loading history are represented in Fig. 7.

The computation is three-dimensional although the problem is plane, since the displacements in z-direction are blocked. A constant Poisson coefficient $v=0.3$ is assumed for the elasto-plastic and elasto-visco-plastic law. A constant pressure $p$ is applied totally for $\mathrm{t}=$ 0 seconds in the lateral faces and the imposed displacement varies linearly from 0 to $2.5 \mathrm{~cm}$ in 120 seconds, corresponding to a deformation rate of $0.208 \mathrm{~mm} / \mathrm{s}$, has shown in Fig. 7. For the elasto-plastic law $\alpha=0.3, k=12 M P a, E^{\prime}=20600 \mathrm{Mpa}$, where assumed. The relaxation parameter and the elastic modulus considered in the visco-elastic components are $\beta=16 \mathrm{~s}^{-1}$ and $E=20600 M P a$, respectively. 


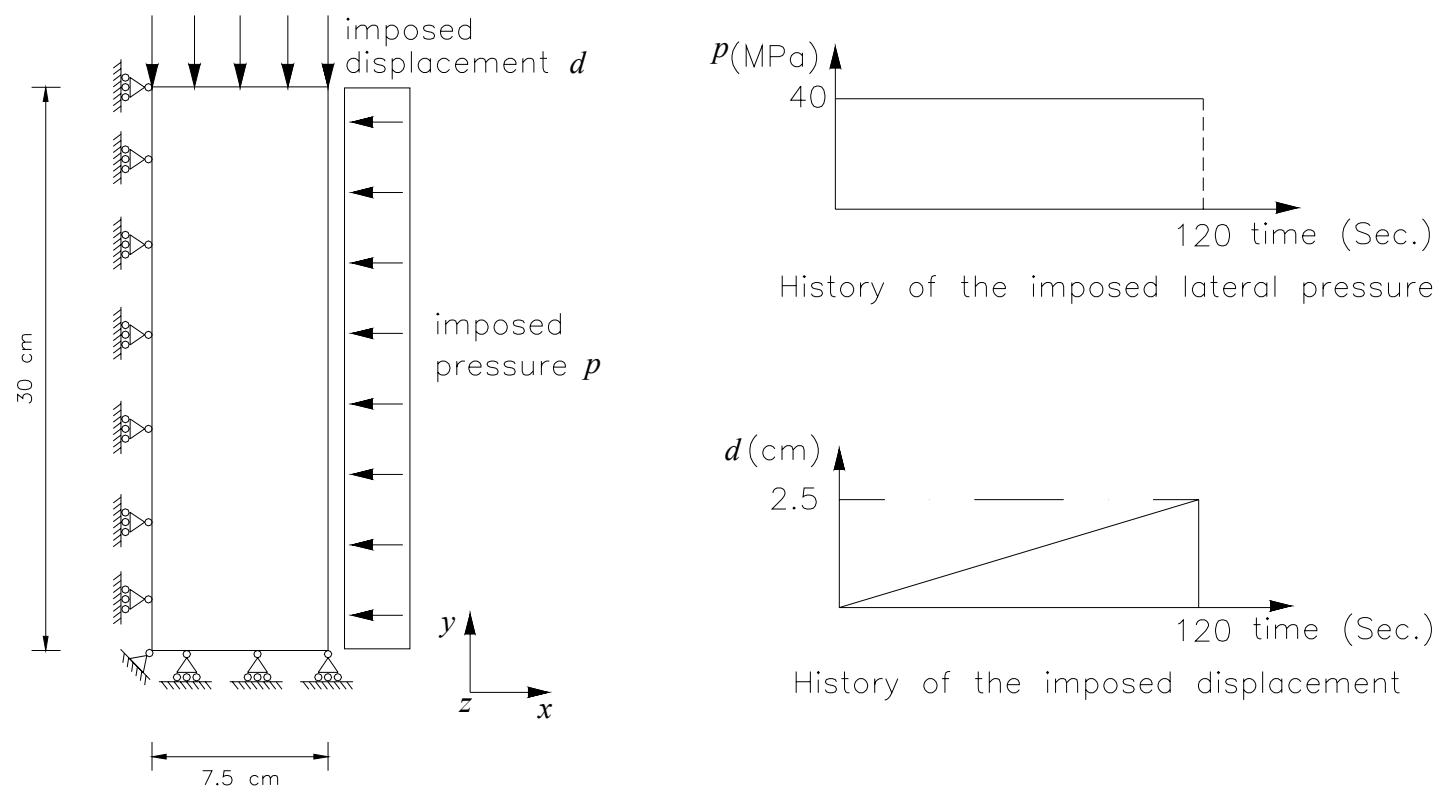

Fig. 7 Specimen geometry, boundary conditions and loading history.

The formation of shear bands and stress concentration is triggered considering a non prismatic shape for the specimen, at mid-height the width of the specimen is equal to $74 \mathrm{~mm}$ and linearly extended to both ends of the specimen, Fig. 7.

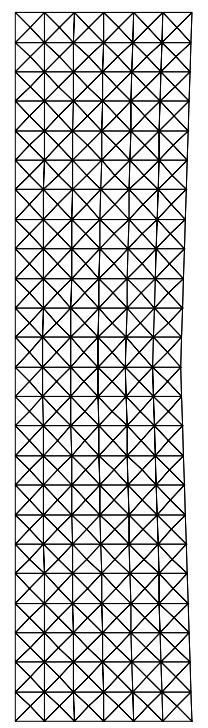

Mesh 1, (144 PT6E elements)

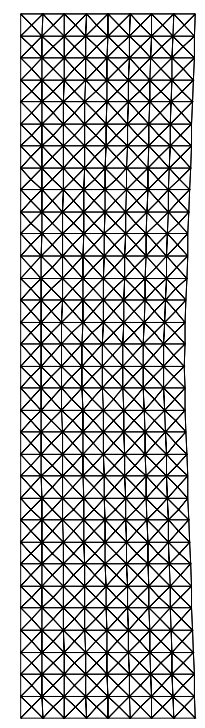

Mesh 2, (256 PT6E elements)

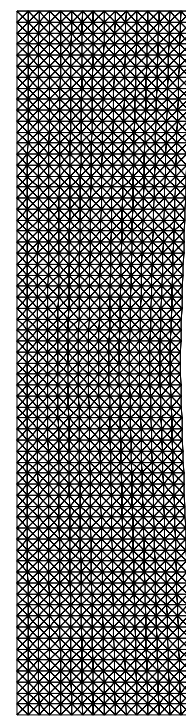

Mesh 3, (1024 PT6E elements)

Fig. 8 Meshes used.

When the dynamic effects (inertial forces) are not considered and the viscosity is assumed equal to zero $(\beta=\infty)$, it is not possible to capture the formation of shear bands. For this reason a 'less regularized' solution, with $\beta=60000 \mathrm{~s}^{-1}$, for each mesh, was first computed and obtained results are presented in Fig. 9. In this situation the computations were carried out until the collapse of the numerical computation. 


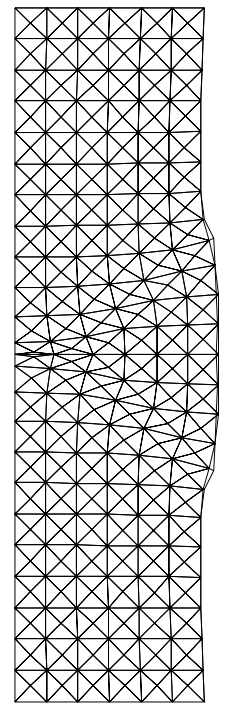

$\mathrm{t}=93.50$ seconds Mesh 1

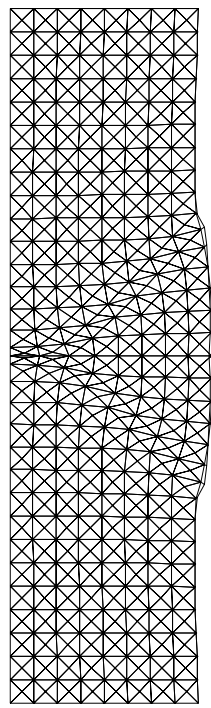

$\mathrm{t}=88.08$ seconds Mesh 2

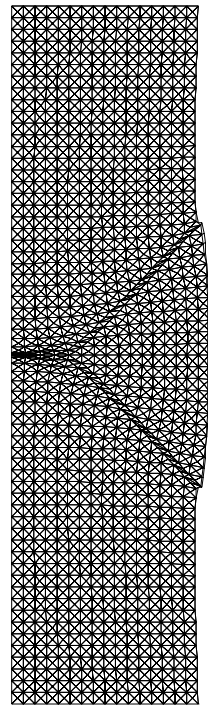

$\mathrm{t}=84.96$ seconds Mesh 3

Fig. 9 Deformed meshes at he moment of the computational collapse, and with low viscosity $\beta=60000 \mathrm{~s}^{-1}$.

It is observed the sharp localization of plastification within a narrow band containing not more than 2 elements in the thickness. Furthermore, it was not possible to apply all the imposed displacement since calculations stops due to numerical collapse. It can be observed that, both the deformed shape and the time of computational collapse are quite different in the three meshes.

In the computations with regularization, the value of the relaxation modulus $\beta$ was gradually decreased $\left(\beta=16 \mathrm{~s}^{-1}\right)$ until a shear band with more than one element in the width was obtained. In the following figures (Figs. 10 and 11) the numerical elasto-plastic solution is regularized with a visco-elastic procedure.

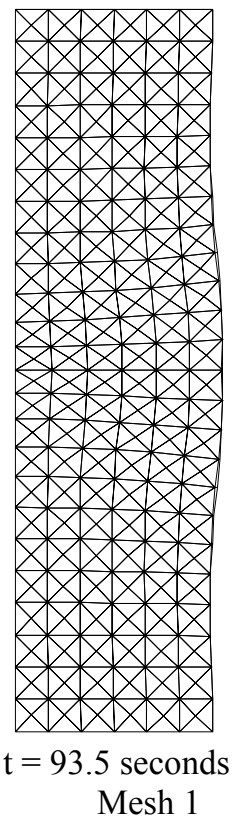

Mesh 1

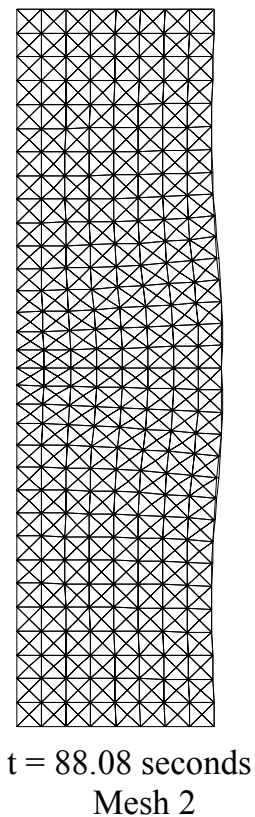

Mesh 2

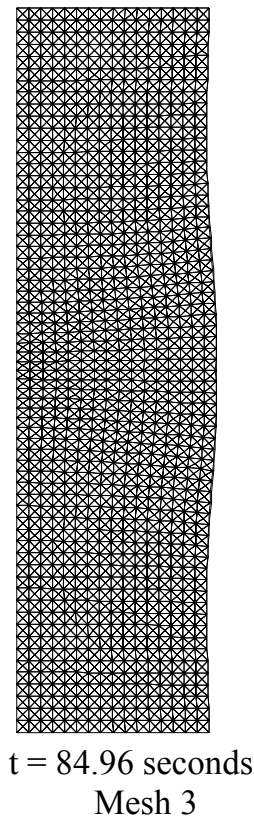

Mesh 3

Fig. 10 Deformed meshes, with high viscosity $\beta=16 \mathrm{~s}^{-1}$. 

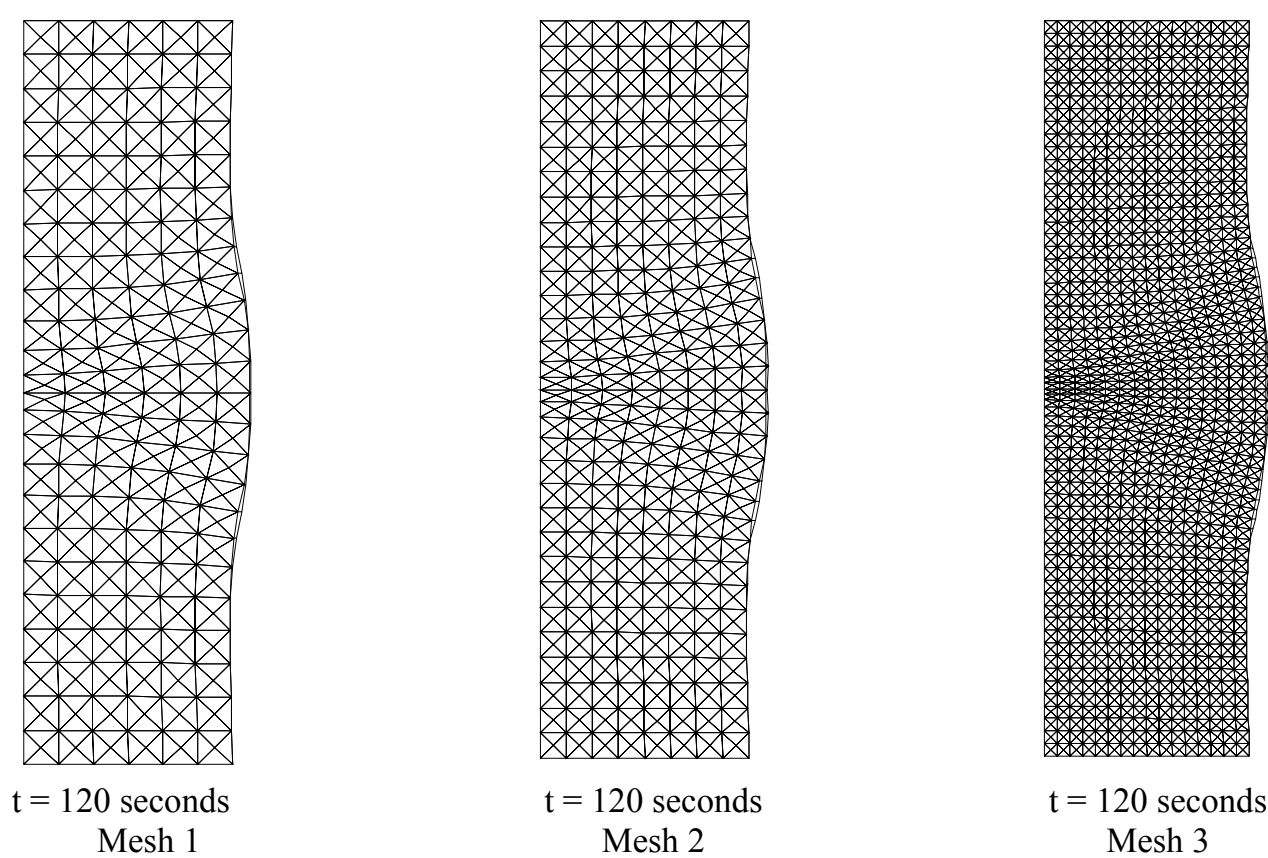

Fig. 11 Deformed meshes for $\mathrm{t}=120$ seconds, $\beta=60 \mathrm{~s}^{-1}$.

\section{CONCLUDING REMARKS}

Viscoelasticity has been introduced as a procedure to regularize the elasto-plastic solution, when strain softening occurs. The procedure adopted is general and therefore has the advantage of allowing the regularization of any elasto-plastic material. Stable and convergent solutions are obtained. In fact, the bands of intense straining appear latter and the dependence on the mesh refinement is strongly reduced.

It is remarked that, for mesh refinement the elasto-plastic problem did not allow to apply the total load without numerical collapse, Fig. 9. The bigger the mesh refinement bigger is the band of straining, which also occur sooner. Increasing the mesh refinement, the band of straining enlarges and occurs sooner

The procedure to obtain bands of intense straining is computationally expensive. For the mesh 3 it was necessary 20 hours of computations $(1.5 \mathrm{GHz}$ processor) to obtain the results corresponding to the last load increment

An efficient implicit time stepping algorithm was used to advance the solution in time. An unconditionally stress point algorithm was used to integrate the elasto-plastic constitutive equations. Therefore, the most important numerical restriction of the proposed computational procedure is introduced by the relaxation module $\beta$, which controls the amplitude of the regularization. The shear band widths depend crucially on the viscosity. A sufficiently high value the viscosity parameter is used, the value $\beta=16 \mathrm{sec}^{-1}$ has made possible to obtain good results for the three considered meshes.

In the FE-computations a total lagrangian formulation was used, being assumed that the used constitutive laws represent the relation between Piola-Kirchhoff stresses and Green strains. 


\section{REFERENCES}

[1] V. D. Silva, F. Casadei, An implementation of the Cam-Clay elasto-plastic model using a backward interpolation and visco-Plastic regularization, Technical Note N. ${ }^{\circ}$ I.96.239, Institute for Systems, Informatics and Safety, Joint Research Centre, Ispra, Italy, 1996.

[2] J. Argyris, I. St. Doltsinis, V. D. Silva, Constitutive modelling and computation of nonlinear viscoelastic solids - Part I: Rheological Models and Numerical Integration Techniques. Computer Methods in Applied Mechanics and Engineering, Vol. 88 No.2, pp. 135-163, 1991.

[3] V. D Silva, A simple model for viscous regularization of elasto-plastic constitutive laws with softening. Communications in Numerical Methods in Engineering, John Wiley \& Sons, Ltd., Vol. 20, pp. 547-568, 2004.

[4] V. D Silva, Viscoplastic regularization of a Cam-Clay FE-Implementation, ECCM'99, European Conference on Computational Mechanics, 31 August- 3September 1999, München, paper $n^{\circ} 442$.

[5] V. D. Silva, Mechanics and Strength of Materials, ISBN 3-540-25131-6, 529 p., 402 illus., Springer, 2006.

[6] R. Cardoso, Regularização visco-elástica de problemas elasto-plásticos com amaciamento, MS.c Thesis, University of Coimbra, 2001 (in Portuguese).

[7] H. Varum, R. Cardoso, A geometrical non-linear model for cable systems analysis, Second International Conference on Textile Composites and Inflatable Structures, Eds. E. Onãte and B. Kröplin, Stuttgart 2-5 October 2005, pp. 234-242.

[8] M. Ortiz, E. P. Popov, Accuracy and stability of integration algorithms for elasto-plastic constitutive relations, International Journal For Numerical Methods in Engineering, Vol. 21, pp. 1561-1576, 1986.

[9] B. Loret, J. H. Prevost, Accurate numerical solution for Drucker-Prager elastic-plastic models. Computer Methods in Applied Mechanics and Engineering, Vol. 54, pp. 259-277 North Holland, 1986.

[10] B. Loret, J. H. Prevost, Dynamic strain localization in elasto-(visco)-plastic Solids, Part 1. General formulation and one-dimensional examples. Computer Methods in Applied Mechanics and Engineering, Vol. 83, pp. 247-273 North Holland, 1990.

[11] A. Needleman, Material rate dependence and mesh sensivity in localization problems. Computer Methods in Applied Mechanics and Engineering, Vol. 67, pp. 69-85 North Holland, 1988.

[12] G. C. Nayak, O. C. Zienkiewicz, Elasto-plastic stress analysis. A generalization for various constitutive relations including strain softening. International Journal for Numerical Methods in Engineering, Vol 5, pp. 113-135, 1972.

[13] H. Wüstenberg, FEPS 3.3- Finite Element Programming System, Element Library. ICA Report No. 22. Stuttgart, 1986.

[14] O. C. Zienkiewicz, R. L. Taylor, The Finite Element Method, Volume 2, Fourth Edition, McGraw-Hill, London, 1991. 